\title{
ACEITAÇÃO DA DOR: ABORDAGEM PSICOMÉTRICA DO CHRONIC PAIN ACCEPTANCE QUESTIONNAIRE NUMA AMOSTRA PROTUGUESA COM DOR CRÓNICA
}

\author{
Joana Costa ${ }^{1}$ \\ José Pinto Gouveia ${ }^{2}$
}

Resumo: O estudo apresenta as características psicométricas da versão portuguesa do Chronic Pain Acceptance Questionnaire (CPAQ: McCracken, Vowles \& Eccleston, 2004; tradução e adaptação Pinto Gouveia \& Costa, 2008), um questionário de auto-relato que avalia a disponibilidade para a experiência da dor e para a realização de actividades, numa amostra de 104 adultos com dor crónica. Foi obtida uma estrutura de dois factores que explicou $51 \%$ da variância. A estrutura factorial obtida foi concordante com o modelo original e apresentou valores de fidelidade e validade adequados. O estudo da validade convergente evidenciou as relações esperadas entre aceitação da dor, psicopatologia, auto-compaixão, evitamento experiencial e ruminação, reforçando o contributo desta medida na explicação dos sintomas de depressão, ansiedade e stress. No âmbito da validade divergente, os resultados obtidos permitiram salientar a capacidade da CPAQ para discriminar os indicadores de psicopatologia.

Os resultados obtidos fornecem suporte empírico aos modelos conceptuais baseados na Acceptance and Commitment Therapy (ACT: Hayes, Strosahl \& Wilson, 1999), e reforçam a literatura existente sobre a sua aplicabilidade à dor crónica.

Palavras-chave: aceitação da dor, ACT, psicopatologia, dor crónica.

Pain acceptance: Psychometric approach to the Chronic Pain Acceptance Questionnaire in a Portuguese sample with chronic pain (Abstract): The present study presents the psychometric properties of the Portuguese version of The Chronic Pain Acceptance Questionnaire (McCracken, Vowles \& Eccleston, 2004; translated and adapted by Pinto Gouveia \& Costa, 2008). This self-report questionnaire assesses the willingness to experience pain and life activities regardless of pain, in 104 Portuguese adults with chronic pain. Factor analysis showed a two factors structure which explained $51 \%$ of the total variance. The structure was similar to the original model and had adequate reliability. Convergent validity supported the expected relations between pain acceptance, psychopathology, self

\footnotetext{
${ }^{1}$ CINEICC - Faculdade de Psicologia e de Ciências da Educação, Universidade de Coimbra.

${ }^{2}$ Faculdade de Psicologia e de Ciências da Educação, Universidade de Coimbra.
} 
compassion, experiential avoidance and rumination and supported the contribution of this construct in explaining depression, anxiety and stress. The results obtained also contributed for the discriminative power of this measure. Moreover, results offered empirical support to conceptual models based on the Acceptance and Commitment Therapy (ACT: Hayes, Strosahl \& Wilson, 1999) and reinforced the existing literature about its application to chronic pain.

Key-words: pain acceptance, ACT, psychopathology, chronic pain.

Ao longo das últimas décadas, a literatura tem identificado múltiplos efeitos da dor crónica no funcionamento físico, psicológico e social, salientando o impacto dos conteúdos cognitivos dos pacientes na exacerbação ou na manutenção da dor, nos comportamentos relacionados com a dor, no mau ajustamento, na procura de cuidados de saúde e na resposta aos tratamentos (Dimond \& Coniam, 1997; Evers, Kraaimaat, Riel \& Jong, 2002; Flor \& Turk, 1988; Schiaffiano, Scaworyn \& Blum, 1998; Smith \& Wallston, 1992; Turk \& Okifuji, 2002). É aceite na literatura que a forma de lidar com a doença requer que o indivíduo doente direccione a sua actividade cognitiva para a preservação da sua integridade física e psicológica, alterando a forma como se percepciona a si próprio e ao ambiente social que o rodeia. A interpretação da dor como algo incontrolável pressupõe que o indivíduo direccione a sua atenção para uma potencial ameaça ao bem-estar, podendo amplificar a sua intensidade e a interferência no funcionamento, ao evitar as actividades que associa ao aumento deste sintoma (Burns, Kubilus, Bruehl, Harden \& Lofland, 2003; Jensen, Turner, Romano \& Lawler, 1994).

Para as abordagens baseadas na Acceptance and Commitment Therapy (ACT: Hayes, Strosahl \& Wilson, 1999), a dor é conceptualizada como parte inevitável da vida. Quando esta realidade é recusada e se luta para a evitar, há um aumento do sofrimento, sob a forma de stress, ansiedade e depressão. Assim, tentativas continuadas de evitar, controlar ou modificar a dor, revelam-se disruptivas, na medida em que promovem o afastamento de um funcionamento adaptativo. De acordo com a ACT, as tentativas de controlo da experiência dolorosa, prevalentes muitas vezes pela busca continuada de novos tratamentos/soluções, promovem o aumento do sofrimento e afastam o indivíduo do momento presente, dos objectivos valorizados e de um funcionamento adaptativo. Deste modo, o que torna uma determinada experiência interna disfuncional são as tentativas de evitar, controlar ou modificar os pensamentos, sentimentos e sensações associados, e não os pensamentos, sentimentos ou sensações em si. As abordagens contextualmente baseadas têm assim por objectivo alterar a função dos pensamentos disfuncionais, como são experienciados e como influenciam outros compor- 
tamentos, em vez de tentar alterar a sua forma ou frequência (Bach \& Moran, 2008; Baer, 2006; Dahl, Wilson, Luciano \& Hayes, 2005; Dahl, Wilson \& Nilsson, 2004; Hayes \& Duckworth, 2006; Hayes et al., 1999; McCracken \& Eccleston, 2005; McCracken, Vowles \& Eccleston 2004; McCracken \& Vowles, 2007; Viane, Crombez, Eccleston, Poppe, Devulde, Van Houdenhove et al., 2003; Wicksell, Mellin \& Olsson, 2007).

Como intervenção psicológica, a ACT é baseada na Relational Frame Theory (RFT: Hayes citado por Hayes et al., 1999), uma teoria compreensiva da linguagem e da cognição, segundo a qual a linguagem humana amplia o conjunto de situações aversivas, porque o comportamento simbólico permite a categorização dos acontecimentos privados e o contacto com eles em múltiplos contextos (Hayes et al., 1999; Hayes, Strosahl, Wilson, Bissett, Psitorello, Toarmino et al., 2004). De acordo com a perspectiva da $\mathrm{ACT} / \mathrm{RFT}$, a psicopatologia resulta da forma como a linguagem e a cognição interagem perante contingências directas, produzindo uma inabilidade em persistir ou mudar o comportamento, em função dos objectivos valorizados. Este tipo de inflexibilidade psicológica emerge de défices no controlo contextual dos processos de linguagem. Ao enfatizar a diferença entre a forma e a função dos pensamentos, comportamentos e sensações indesejados, as abordagens baseadas na ACT introduzem um aspecto inovador no seio da psicologia. Assim, a problemática não é o facto do indivíduo pensar algo errado, mas sim a sua resposta face a esses pensamentos e a utilização excessiva da linguagem, como estratégia de regulação comportamental (Dahl, et al., 2005; Dahl et al., 2004; Hayes \& Duckworth, 2006; Hayes et al., 1999; Segal, Williams \& Teasdale, 2002). A ACT baseia-se numa análise comportamental que facilita a mudança da função das experiências internas indesejadas. Contrariamente às terapias de primeira e de segunda gerações, o objectivo principal da ACT não é o manejo dos sintomas, mas uma promoção eficaz do repertório de resposta face à experiência de dor, tendo como base os objectivos de vida valorizados, identificados e definidos pelo indivíduo. De acordo com a ACT, a possibilidade de viver uma vida com significado e em conformidade com os objectivos estabelecidos centra-se na disponibilidade do indivíduo para a experiência de dor e na realização de actividades, para além da dor e do mal-estar associado. Viver em conformidade com os objectivos de vida valorizados resulta de um compromisso estabelecido e do conhecimento de que as dificuldades na vida são algo de inevitável (Bach \& Moran, 2008; Dahl et al., 2005; Hayes, Luoma, Bond, Masuda \& Lillis, 2006; Hayes \& Duckworth, 2006; Hayes et al., 1999; Hayes, 2004; Hofmann \& Asmundson, 2007).

No contexto da dor crónica, as intervenções psicológicas tradicionais baseadas na exposição, são muitas vezes conceptualizadas pelo indivíduo como técnicas aversivas. A ACT tende a promover o desenvolvimento destes procedimentos em três formas distintas, respeitantes ao desenvolvimento 
da exposição, à neutralização da inflexibilidade cognitiva e à clarificação de valores (Dahl et al., 2004; Wicksell, Melin \& Olsson, 2007). Através da utilização de processos de mindfulness, de aceitação, de compromisso e de mudança, a ACT visa promover a flexibilidade psicológica, isto é, a capacidade de contactar com o momento presente e com os pensamentos/ sentimentos/ sensações contidas nesse momento, sem necessidade de se defender. Neste âmbito, destaca-se a importância de exercícios mindfulness, através dos quais é possível colocar o indivíduo com dor na experiência do momento. A prática deste tipo de exercícios revela benefícios, ao possibilitar a exposição a experiências internas anteriormente evitadas, promovendo a neutralização emocional e a capacidade de fazer escolhas (expor-se ou evitar a experiência de dor) (Hayes \& Duckworth, 2006; McCracken, 2006; McCracken, Gauntlett-Gilbert \& Vowles, 2007; McCracken \& Vowles, 2007; Morone, Greco \& Weiner, 2008; Wicksell et al., 2007).

Alguns modelos psicológicos da dor crónica têm sugerido que o indivíduo tende a focar-se na dor, nos aspectos negativos da sua situação e a fixar padrões recorrentes desadaptativos como forma de a tentar controlar. A literatura tem vindo a valorizar a aceitação como constructo determinante na forma como o indivíduo reage e se adapta a situações de dor crónica (McCracken et al., 2004; McCracken et al., 2007).

A aceitação traduz assim uma disponibilidade activa e consciente para experienciar acontecimentos internos indesejados, na busca dos seus próprios valores e objectivos (McCracken, 1998; McCracken \& Eccleston, 2005; McCracken, et al., 2004; McCracken \& Yang, 2006; Viane, Crombez, Eccleston, Poppe, Devulder, et al., 2003). Para McCracken (1998) a aceitação dor passa pela a disponibilidade do indivíduo para aprender a observar experiências internas sem as tentar evitar, modificar ou controlar. Traduz assim um processo activo de sentir os sentimentos como sentimentos, pensar os pensamentos como pensamentos e, actuar eficazmente em conformidade com os valores e objectivos de vida estabelecidos (Bach \& Moran, 2008; Baer, 2006; Blackledge \& Hayes, 2001; Hayes, 2004).

O Acceptance and Action Questionnaire (AAQ: Hayes et al., 2004) foi construído para avaliar a eficácia do modelo baseado na ACT, emergindo da necessidade de desenvolver uma medida geral, breve e aplicável à investigação. A literatura tem feito referência a múltiplos estudos de validação desta medida, existindo várias versões adaptadas a diversos contextos, como por exemplo a Avoidance and Fusion Questionnaire for Youth (Greco, Murrell \& Coyne citado por Bach \& Moran, 2008) que avalia o conjunto dos processos da ACT em jovens e a Body Image Acceptance Questionnaire (Sandoz \& Wilson, citado por Bach \& Moran, 2008) que avalia a aceitação através de sentimentos e pensamentos acerca da forma e peso do corpo.

A Chronic Pain Acceptance Questionnaire (CPAQ: McCracken et al., 2004) traduz a adaptação da medida original ao contexto da dor. Esta 
medida avalia a disponibilidade para a dor e a persistência na realização de actividades consistentes com os objectivos e valores pessoais (Bach \& Moran, 2008; Dahl et al., 2005; McCracken \& Eccleston, 2005; McCracken et al., 2004). São múltiplas as referências encontradas na literatura sobre a importância deste constructo no contexto da dor, estando relacionado com um melhor funcionamento emocional, físico e social, menor recurso a medicação e aos cuidados de saúde. O seu papel preditor no ajustamento à dor crónica tem igualmente sido referido, sendo mesmo considerado como melhor preditor de um funcionamento adaptativo, independentemente da intensidade da dor (Dahl et al., 2005; McCracken, 1998; McCracken \& Eccleston, 2003; McCracken \& Eccleston, 2005; McCracken, Spertus, Janexk, Sinclair \& Wetzel, 1999; McCracken \& Vowles, 2007; McCracken et al., 2004; McCracken \& Yang, 2006; Morone, Greco \& Weiner, 2008; Nicholas \& Asghari, 2006).

A importância da aceitação na resposta fisiológica e no desempenho de tarefas tem igualmente sido ressaltada em estudos experimentais (Campbell-Sills, Barlow, Brown \& Hofmann, 2006; Crombez, Eccleston, DeVlieger, Van Damme \& De Clercq, 2007). Neste sentido, destacam-se os estudos que analisaram a eficácia de intervenções baseadas na aceitação versus intervenções baseadas no controlo cognitivo, que mostraram o papel da aceitação da dor como processo chave da mudança comportamental, ao promover a tolerância à dor e a persistência no comportamento (Gutiérrez, Luciano, Rodríguez \& Fink, 2004; Vowles, McNeil, Gross, McDaniel, Mouse, Bates et al., 2007).

Assim, a presente investigação teve com objectivo analisar as qualidades psicométricas da versão traduzida do Chronic Pain Acceptance Questionnaire (CPAQ: McCracken et al., 2004) numa amostra portuguesa com dor crónica.

\section{Método}

\section{Participantes}

A amostra foi constituída por 104 adultos (40 com diagnóstico de Artrite Reumatóide; 64 com diagnóstico de Dor Crónica sem outra especificação). O Quadro 1 mostra as características demográficas dos participantes. Os participantes foram referenciados pelo médico especialista, sendo uma amostra de conveniência. Apresentaram uma média de idades de 60,81 $(D P=13,24)$, para os homens e de $59,53(D P=14,61)$ para as mulheres. A maioria dos participantes era Casada/União de Facto $(81,7 \%)$, estava reformada $(54.9 \%)$ e apresentava um baixo número de anos de escolaridade. $\mathrm{O}$ estudo das diferenças entre sexos mostrou existirem diferenças significati- 
vas para os anos de escolaridade $(t(62)=-2,464 ; p=0,017)$. A utilização de um teste paramétrico para o estudo das diferenças entre os sexos é justificada pela homogeneidade das variâncias das variáveis em estudo, para além do teste $t$ ser particularmente robusto a violações da normalidade.

Quadro 1. Caracterização demográfica da amostra por sexos

\begin{tabular}{|c|c|c|c|c|c|c|}
\hline & \multicolumn{2}{|c|}{$\begin{array}{c}\text { Sexo Masculino } \\
(\mathrm{N}=21)\end{array}$} & \multicolumn{2}{|c|}{$\begin{array}{c}\text { Sexo Feminino } \\
(\mathbf{N}=83)\end{array}$} & \multirow[b]{2}{*}{$\chi^{2}$} & \multirow[b]{2}{*}{$p$} \\
\hline & $\mathbf{N}$ & $\%$ & $\mathbf{N}$ & $\%$ & & \\
\hline Estado civil & & & & & 4,496 & 0,213 \\
\hline Solteiro & 0 & 0 & 4 & 4.9 & & \\
\hline Casado/ Outro & 21 & 100 & 67 & 81,7 & & \\
\hline Separado/ Divorciado & 0 & 0 & 2 & 2,4 & & \\
\hline Viúvo & 0 & 0 & 9 & 11 & & \\
\hline Profissão & & & & & 1,739 & 0,419 \\
\hline Empregada & 12 & 57,1 & 35 & 42,7 & & \\
\hline Desempregado & 0 & 0 & 2 & 2,4 & & \\
\hline \multirow[t]{3}{*}{ Reformado } & 9 & 42,9 & 45 & 54,9 & & \\
\hline & \multicolumn{2}{|c|}{ Sexo Masculino } & \multicolumn{2}{|c|}{ Sexo Feminino } & & \\
\hline & $\mathbf{M}$ & DP & $\mathbf{M}$ & DP & $t$ & $p$ \\
\hline Idade & 60,81 & 13,235 & 59,53 & 14,608 & 0,333 & 0,740 \\
\hline Habilitacões literárias & 3,60 & 1,595 & 5,25 & 4,224 & $-2,464$ & 0,17 \\
\hline
\end{tabular}

\section{Instrumentos}

Folha de dados Demográficos e Clínicos. Foram registados o sexo, o estado civil, a idade, a situação profissional, as habilitações literárias e o diagnóstico clínico.

Questionário de Aceitação da Dor Crónica (Chronic Pain Acceptance Questionnaire: CPAQ; McCraken, Vowles \& Eccleston, 2004; tradução e adaptação por Pinto Gouveia \& Costa, 2008, manuscrito em preparação), é um questionário de auto-preenchimento, com 20 itens, organizados em duas sub-escalas, (Disposição para a dor: 9 itens; Realização de Actividades:11 itens), com um formato de resposta de tipo Likert de 7 pontos $(0=$ Nunca, a 6 = Sempre), em que os resultados mais elevados significam maior Aceitação da Dor. A medida permite obter resultados totais e resultados parciais; a amplitude de resultados varia entre 0 e 120 (escala total), 0 e 54 (Disposição para a dor) e, entre 0 e 66 (Realização de Actividades). As duas escalas apresentam valores elevados de consistência interna (alfa de Cronbach de 0,82 e 0,78 , respectivamente) e valores aceitáveis de correlação entre as escalas $(0,36)$ (McCraken et al., 2004). No que respeita à validade, 
o estudo com a amostra de aferição obteve uma estrutura com quatro factores (Realização de Actividades, Disponibilidade, Controlo e Cronicidade). No entanto, McCraken et al. (2004) optaram por retirar os dois factores menores, dado apresentarem valores de fidelidade marginais, não estarem relacionados com o valor total da Aceitação nem com outras medidas de mal-estar relacionado com a dor e a incapacidade. A validade foi demonstrada pelas associações encontradas entre a Aceitação da Dor, a intensidade da dor, a procura dos cuidados médicos, a utilização de medicação, o funcionamento físico e questões psicossociais.

Escalas de Ansiedade Depressão e Stress (Depression Anxiety Stress Scales; EADS-42, Lovibond \& Lovibond, 1995; tradução e adaptação por Pais-Ribeiro, Honrado \& Leal, 2004a), são escalas de auto-preenchimento com 42 itens, organizados em três sub-escalas com 14 itens cada (Depressão, Ansiedade e Stress) e formato de resposta de tipo Likert de 5 pontos $(0=$ Não se aplicou nada a mim; 4= Aplicou-se a mim a maior parte das vezes), em que um resultado mais elevado traduz estados emocionais mais negativos A medida permite obter resultados parciais para cada uma das sub-escalas, com uma amplitude de resultados a variar entre 0 e 42 . Apresenta valores elevados de consistência interna (alfa de Cronbach entre 0,83 e 0,93) (Pais-Ribeiro, Honrado \& Leal, 2004a). A validade foi demonstrada pelas associações encontradas entre o item e a escala de pertença e entre o item e a escala a que não pertence (Pais-Ribeiro, Honrado \& Leal, 2004a). Com a amostra do nosso estudo, foram obtidos valores de consistência interna de 0,59 (Ansiedade), 0,95 (Depressão) e 0,91 (Stress).

Questionário de Aceitação e Acção (Acceptance and Action Questionnaire-II: AAQII; Hayes et al., 2004; tradução e adaptação por Pinto Gouveia \& Gregório, 2007, manuscrito em preparação), é um questionário de auto-relato que mede o evitamento experiencial. A medida é composta por 10 itens, com um formato de resposta de tipo Likert de 7 pontos $(1=$ Nunca Verdadeiro; $7=$ Sempre Verdadeiro), em que um resultado mais elevado traduz mais tentativas de evitamento/controlo das experiências internas aversivas. A medida permite obter um resultado global, que varia entre 10 e 70. Apresenta um valor elevado de consistência interna (alfa de Cronbach 0,70), e valores de fidelidade teste-reteste de 0,64 (período de 4 meses entre teste e reteste) (Block-Lerner, Salters-Pedneault \& Tull, 2005; Hayes et al., 2004). A validade convergente e discriminante foi demonstrada pelas relações entre a medida e a evitação, supressão de pensamento, psicopatologia (ansiedade, depressão, stress laboral, sintomatologia pós-traumática), sintomas físicos, bem-estar, qualidade e satisfação com a vida (Block-Lerner et al., 2005; Hayes et al., 2004). Com a amostra do nosso estudo foi obtido um valor de consistência interna elevado (alfa de Cronbach 0,92).

Escala de Auto-Compaixão (Self-Compassion Scale: SELFCS: Neff, 2003; tradução e adaptação: Pinto Gouveia \& Castilho, 2006, manuscrito 
em preparação), é uma escala de auto-relato constituída por 26 itens, organizados em seis sub-escalas designadas Bondade, Auto-Ajuizamento, Humanidade Comum, Isolamento, Mindfulness e Sobre-Identificação, com formato de resposta de tipo Likert de 5 pontos (1. Quase nunca; 5. Quase sempre), em que um resultado mais elevado significa mais auto-compaixão. A medida permite obter um resultado total e resultados parciais, que variam entre um e cinco. Apresenta um valor de consistência interna de 0.92 , para a escala total (Neff, 2003). Para Neff (2003), as associações entre esta medida e a ansiedade, depressão, satisfação com a vida e a auto-estima, forneceram evidência da validade convergente e divergente da medida.

Com a amostra do nosso estudo foi obtido um valor elevado de consistência interna (alfa de Cronbach 0.95).

\section{Procedimento}

Os participantes foram referenciados pelos médicos assistentes dos Centros de Saúde de Torres Vedras, Lourinhã e Cadaval. Todas as informações relacionadas com os objectivos do estudo foram fornecidas aos pacientes, e obtido o consentimento informado para a participação. Os protocolos foram administrados individualmente, num gabinete das respectivas instituições. Todas as dúvidas relacionadas com as questões ou com o preenchimento do questionário foram esclarecidas pelo autor da investigação.

\section{Adaptação da medida à língua portuguesa}

Após contacto com Lance McCraken, autor responsável pela medida original, foi pedida a autorização para a utilização e tradução da CPAQ para a língua portuguesa. Os itens foram traduzidos da língua inglesa para a língua portuguesa por um psicólogo que domina a língua inglesa escrita e falada, sendo verificados aspectos relativos à semelhança lexical e conceptual, procurando preservar o conteúdo de cada item. No que respeita a tradução e adaptação do item 1 "I am getting on with the business of living no matter what my level of pain is", optou-se por preservar o conteúdo inicial ("Estou a entender-me com os meus negócios de vida independentemente do nível de dor"), apesar da semelhança lexical suscitar algumas controvérsias. Na língua portuguesa não é utilizada a expressão “...entender-me com os meus negócios de vida...", sendo perceptível o significado "Estou a entender-me com a minha vida". A retroversão da medida foi realizada por outro psicólogo com formação na língua inglesa. As duas versões foram comparadas e ajustados os parâmetros discordantes, mantendo os aspectos implícitos à construção da medida original. No Quadro 2 são apresentados os itens que constituem as versões originais das medidas, em inglês, e as correspondentes versões traduzidas para a língua portuguesa. 
Quadro 2. Correspondência dos itens da CPAQ traduzidos para a aferição portuguesa e os itens da versão original.

\section{Versão traduzida para a língua Por- Versão original em língua Inglesa} tuguesa

1. Estou a entender-me com os meus negócios (de vida) independentemente do nível de dor

2. A minha vida corre bem, embora tenha uma dor crónica

3. Não há problema em ter dor

4. Sacrificaria com muito prazer coisas importantes na minha vida para controlar melhor esta dor

5. Não é necessário controlar a minha dor para continuar com a minha vida

6. Embora as coisas tenham mudado, levo uma vida normal apesar da minha dor crónica

7. Preciso de me concentrar para me livrar da minha dor

8. Existem muitas actividades que faço quando estou com dor

9. Tenho uma vida completa apesar da dor crónica.

10. Controlar a dor não é um objectivo importante na minha vida

11. Preciso mudar os meus pensamentos e sentimentos acerca da dor, para conseguir dar passos importantes na minha vida

12. Apesar da dor, mantenho os rumos que escolhi para a minha vida

13. Ter a dor num nível controlado assume prioridade sempre que estou a fazer alguma coisa

14. Antes de fazer qualquer plano sério, tenho de ter algum controlo na minha dor

15. Quando a dor aumenta, consigo tomar conta das minhas responsabilidades

16. Terei um melhor controlo na minha vida se conseguir controlar os pensamentos negativos acerca da dor
1. I am getting on with the business of living no matter what my level of pain is

2. My life is going well, even though I have chronic pain

3. It's O.K. to experience pain

4. I would gladly sacrifice important things in my life to control this pain better

5. It's not necessary for me to control my pain in order to handle my life well

6. Although things have changed, I am living a normal life despite my chronic pain

7. I need to concentrate on getting rid of my pain

8. There are many activities I do when I feel pain

9. I lead a full life even though I have chronic pain

10. Controlling pain is less important than other goals in my life

11. My thoughts and feelings about pain must change before I can take important steps in my life

12. Despite the pain, I am now sticking to a certain course in my life

13. Keeping my pain level under control takes first priority whenever I am doing something

14. Before I can make any serious plans, I have to get some control over my pain

15. When my pain increases, I can still take care of my responsibilities

16. I will have better control over my life if I can control my negative thoughts about pain 
17. Evito colocar-me em situações nas quais a dor pode aumentar

18. As minhas preocupações e receios acerca do que a dor me irá fazer, são verdadeiros

19. É um alívio perceber que não tenho de mudar/ alterar a minha dor para continuar com a minha vida

20. Tenho de lutar muito/ trabalhar muito para fazer as coisas quando estou com dor
17. I avoid putting myself in situations where pain might increase

18. My worries and fears about what pain will do to me are true

19. It's a relief to realize that I don't have to change my pain to get on with my life

20. I have to struggle to do things when I have pain

\section{Resultados}

Os aspectos relativos à normalidade e homogeneidade das variáveis foram analisados através do teste de Kolmogorov-Smirnov e do teste de Shapiro-Wilk, o enviesamento em relação à média através dos valores de Skewness, e o coeficiente de achatamento através dos valores de Kurtosis. Os pressupostos da normalidade foram garantidos para a sub-escala de stress, mas não para as sub-escalas de ansiedade e de depressão, apesar de não se afastarem muito da distribuição normal. Utilizámos testes paramétricos, pela robustez que apresentam face a violações à normalidade das variáveis. Segundo Maroco (2007) à medida que a amostra aumenta, a distribuição vai assumindo a forma de distribuição normal, ainda que a distribuição da variável seja enviesada, sendo assumida a distribuição da média amostral satisfatoriamente próxima da normal.

A análise de outliers evidenciou a existência de um valor extremo para a ansiedade e alguns outliers para a depressão. Os resultados foram analisados com e sem a presença destes valores extremos, não se verificando diferenças significativas.

\section{Validade factorial do Chronic Pain Acceptance Questionnaire}

Os valores do Kaiser-Meyer-Olkin Measure of Sampling $(0,872)$ e do Bartlett's Test of Sphericity $(1103,586 ; p=0,000)$, permitiram a realização da Análise Factorial em Componentes Principais aos 20 itens, com rotação oblíqua (Oblimin). Pela análise dos dados da distribuição (screen-plot) e com o critério de retenção dos valores próprios (eigenvalues) superiores a 1 , testaram-se as soluções factoriais com 2, 3 e 4 factores. Foi adoptada a solução de dois factores, que permitiu explicar 50,741\%, com 20 itens. 
Quadro 3. Correspondência dos itens da CPAQ aos factores obtidos na Análise Factorial em Componentes Principais.

\begin{tabular}{|c|c|c|}
\hline \multirow[b]{2}{*}{ ITEMS } & \multicolumn{2}{|c|}{ Factor } \\
\hline & 1 & 2 \\
\hline CPAQ_9 - Tenho uma vida completa apesar da dor crónica & $\mathbf{0 , 8 5 2}$ & 0,221 \\
\hline $\begin{array}{l}\text { CPAQ_2 - A minha vida corre bem, embora tenha uma dor } \\
\text { crónica }\end{array}$ & $\mathbf{0 , 8 4 1}$ & 0,172 \\
\hline $\begin{array}{l}\text { CPAQ_1 - Estou a entender-me com os meus negócios (de } \\
\text { vida) independentemente do nível de dor }\end{array}$ & $\mathbf{0 , 8 2 5}$ & 0,341 \\
\hline $\begin{array}{l}\text { CPAQ_6 - Embora as coisas tenham mudado, levo uma vida } \\
\text { normal apesar da minha dor crónica }\end{array}$ & $\mathbf{0 , 8 2 0}$ & 0,242 \\
\hline $\begin{array}{l}\text { CPAQ_ } 8 \text { - Existem muitas actividades que faço quando estou } \\
\text { com dor }\end{array}$ & 0,786 & 0,348 \\
\hline $\begin{array}{l}\text { CPAQ_12 - Apesar da dor, mantenho os rumos que escolhi para } \\
\text { a minha vida }\end{array}$ & $\mathbf{0 , 7 8 5}$ & 0,299 \\
\hline $\begin{array}{l}\text { CPAQ_15 - Quando a dor aumenta, consigo tomar conta das } \\
\text { minhas responsabilidades }\end{array}$ & 0,708 & 0,336 \\
\hline $\begin{array}{l}\text { CPAQ_5 - Não é necessário controlar a minha dor para conti- } \\
\text { nuar com a minha vida. }\end{array}$ & $\mathbf{0 , 6 3 5}$ & 0,453 \\
\hline $\begin{array}{l}\text { CPAQ_ } 4 \text { - Sacrificaria com muito prazer coisas importantes na } \\
\text { minha vida para controlar melhor esta dor }\end{array}$ & 0,475 & 0,337 \\
\hline $\begin{array}{l}\text { CPAQ } 19 \text { - É um alívio perceber que não tenho de mudar/ } \\
\text { alterar a minha dor para continuar com a minha vida }\end{array}$ & $\mathbf{0 , 4 5 8}$ & - \\
\hline $\begin{array}{l}\text { CPAQ_10 - Controlar a dor não é um objectivo importante na } \\
\text { minha vida }\end{array}$ & 0,416 & 0,320 \\
\hline $\begin{array}{l}\text { CPAQ_17-Evito colocar-me em situações nas quais a dor pode } \\
\text { aumentar }\end{array}$ & $\mathbf{0 , 1 3 0}$ & - \\
\hline $\begin{array}{l}\text { CPAQ_11 - Preciso mudar os meus pensamentos e sentimentos } \\
\text { acerca da dor, para conseguir tomar passos importantes na } \\
\text { minha vida }\end{array}$ & 0,198 & 0,876 \\
\hline $\begin{array}{l}\text { CPAQ_16 - Terei um melhor controlo na minha vida se conse- } \\
\text { guir controlar os pensamentos negativos acerca da dor }\end{array}$ & 0,233 & $\mathbf{0 , 8 4 3}$ \\
\hline $\begin{array}{l}\text { CPAQ_7 - Preciso de me concentrar para me livrar da minha } \\
\text { dor }\end{array}$ & 0,136 & $\mathbf{0 , 8 1 0}$ \\
\hline $\begin{array}{l}\text { CPAQ_13 - Ter a dor num nível controlado assume prioridade } \\
\text { sempre que estou a fazer alguma coisa }\end{array}$ & 0,382 & 0,747 \\
\hline $\begin{array}{l}\text { CPAQ_20 - Tenho de lutar muito/ trabalhar muito para fazer as } \\
\text { coisas quando estou com dor }\end{array}$ & 0,558 & 0,714 \\
\hline $\begin{array}{l}\text { CPAQ_14 - Antes de fazer qualquer plano sério, tenho de ter } \\
\text { algum controlo na minha dor }\end{array}$ & 0,493 & 0,556 \\
\hline CPAQ_3 - Não há problema em ter dor & 0,430 & 0,464 \\
\hline $\begin{array}{l}\text { CPAQ_18 - As minhas preocupações e receios acerca do que a } \\
\text { dor me irá fazer, são verdadeiros }\end{array}$ & - & $\mathbf{0 , 4 4 7}$ \\
\hline
\end{tabular}


Os valores das comunalidades mostraram, de um modo geral, a adequação ao modelo obtido. $\mathrm{O}$ item 17 é o que apresenta menor adequação ao modelo. A tabela 3 mostra os pesos dos itens em factor. Em negrito são assinalados os pesos dos itens no factor a que pertencem, na estrutura factorial adoptada. A organização dos itens é semelhante à versão original, com excepção dos itens 3,4 e 17 . Seguindo o mesmo tipo de abordagem dos autores da versão original, os itens discordantes foram individualmente analisados, para inclusão na respectiva sub-escala. Assim, cada sub-escala foi analisada em termos de comportamento da consistência interna, com a inclusão/exclusão destes itens. O item 3 foi incluído no factor 1 , e os itens 4 e 17 foram incluídos no factor 2, ao promoverem aumento do valor de consistência interna e dos valores de correlação item-total. Apesar do item 17 saturar abaixo de 0,10 no factor 2 , optámos por preservar a estrutura da escala original, pela análise do valor de consistência interna do factor caso este item fosse eliminado.

Tendo por base as sugestões de McCraken et al. (2004), e de acordo com os pesos factoriais obtidos, designámos o Factor 1: Realização de Actividades e o Factor 2: Disponibilidade para a dor.

Os factores obtidos mostraram valores elevados de consistência interna, analisados através do alfa de Cronbach (Realização de Actividades: 0,83; Disponibilidade para a dor: 0,89; Escala Total: 0,91). O Quadro 4 mostra os valores da média, desvio padrão, valores das correlações item-total, e de consistência interna da versão final da medida. Entre parênteses constam os valores obtidos para a versão original da medida.

De uma forma geral, a solução de dois factores obtida para a versão traduzida da CPAQ (McCraken et al., 2004) apresenta validade factorial, indicando a distribuição obtida uma sobreposição ao padrão original. Os pesos factoriais dos itens são elevados no factor de pertença, com excepção do item 17 ("Evito colocar-me em situações nas quais a dor pode aumentar"). As sub-escalas da versão obtida têm valores de consistência interna superiores ou iguais a 0,83 , e superiores à versão original $(0,89$ e 0,83 , versão obtida; 0,82 e 0,78 , para a versão original).

\section{Validade Convergente}

A validade convergente da CPAQ foi analisada, com recurso a matrizes de correlação, através do coeficiente de correlação de Pearson. Os resultados obtidos revelaram que a Realização de Actividades e a Disponibilidade para a dor apresentaram correlações fracas e negativas com os sintomas psicopatológicos, com valores que variaram entre $-0,277(p=0,007)$, para a relação entre a Disponibilidade para a dor e a Ansiedade, e -0,496 $(p=0,000)$, para a relação entre a Realização de Actividades e a Depressão. 
Quadro 4. Valores médios, de desvio padrão, de correlação item-total e de correlação alfa de Cronbach para os itens da versão final da CPAQ.

\begin{tabular}{|c|c|c|c|}
\hline Designação na Versão portuguesa & $M$ & DP & $\begin{array}{l}\text { Item- } \\
\text {-total }\end{array}$ \\
\hline $\begin{array}{l}\text { Factor } 1 \text { - Realização de Actividades ( } \alpha \text { de Cron- } \\
\text { bach 0,89-0,82) }\end{array}$ & & & $0,34-0,78$ \\
\hline $\begin{array}{l}\text { CPAQ_9 - Tenho uma vida completa apesar da dor } \\
\text { crónica }\end{array}$ & 4,38 & 1,66 & 0,75 \\
\hline $\begin{array}{l}\text { CPAQ } 2 \text { - A minha vida corre bem, embora tenha uma } \\
\text { dor crónica }\end{array}$ & 4,04 & 1,78 & 0,73 \\
\hline $\begin{array}{l}\text { CPAQ } 1 \text { - Estou a entender-me com os meus negócios } \\
\text { (de vida) independentemente do nível de dor }\end{array}$ & 3,89 & 1,70 & 0,78 \\
\hline $\begin{array}{l}\text { CPAQ_6 - Embora as coisas tenham mudado, levo uma } \\
\text { vida normal apesar da minha dor crónica }\end{array}$ & 4,17 & 1,70 & 0,74 \\
\hline $\begin{array}{l}\text { CPAQ_8 - Existem muitas actividades que faço quando } \\
\text { estou com dor }\end{array}$ & 3,57 & 1,61 & 0,72 \\
\hline $\begin{array}{l}\text { CPAQ 12 - Apesar da dor, mantenho os rumos que } \\
\text { escolhi para a minha vida }\end{array}$ & 4,22 & 1,89 & 0,70 \\
\hline $\begin{array}{l}\text { CPAQ_15 - Quando a dor aumenta, consigo tomar conta } \\
\text { das minhas responsabilidades }\end{array}$ & 3,34 & 1,83 & 0,64 \\
\hline CPAQ_5 - Não é necessário controlar a minha dor para & 2,19 & 2,23 & 0,60 \\
\hline $\begin{array}{l}\text { CPAQ } 19 \text { - É um alívio perceber que não tenho de } \\
\text { mudar/ alterar a minha dor para continuar com a minha } \\
\text { vida }\end{array}$ & 5,01 & 1,65 & 0,34 \\
\hline $\begin{array}{l}\text { CPAQ_10 - Controlar a dor não é um objectivo impor- } \\
\text { tante na minha vida }\end{array}$ & 0,51 & 1,38 & 0,40 \\
\hline CPAQ 3 - Não há problema em ter dor & 1,03 & 1,74 & 0,41 \\
\hline $\begin{array}{l}\text { Factor } 2 \text { - Disponibilidade para a dor ( } \alpha \text { de Cron- } \\
\text { bach } 0,89-0,78)\end{array}$ & & & $05-0,76$ \\
\hline $\begin{array}{l}\text { CPAQ_11 - Preciso mudar os meus pensamentos e sen- } \\
\text { timentos acerca da dor, para conseguir tomar passos } \\
\text { importantes na minha vida }\end{array}$ & 2,84 & 2,48 & 0,76 \\
\hline $\begin{array}{l}\text { CPAQ_16 - Terei um melhor controlo na minha vida se } \\
\text { conseguir controlar os pensamentos negativos acerca da } \\
\text { dor }\end{array}$ & 2,26 & 2,48 & 0,72 \\
\hline $\begin{array}{l}\text { CPAQ } 7 \text { - Preciso de me concentrar para me livrar da } \\
\text { minha dor }\end{array}$ & 2,53 & 2,20 & 0,68 \\
\hline $\begin{array}{l}\text { CPAQ_13 - Ter a dor num nível controlado assume } \\
\text { prioridade sempre que estou a fazer alguma coisa }\end{array}$ & 1,03 & 1,58 & 0,64 \\
\hline $\begin{array}{l}\text { CPAQ_20 - Tenho de lutar muito/ trabalhar muito para } \\
\text { fazer as coisas quando estou com dor } \\
\text { CPAO } 14 \text { - Antes de fazer qualquer plano sério tenho }\end{array}$ & 1,70 & 1,93 & 0,65 \\
\hline $\begin{array}{l}\text { CPAQ } 14-\text { Antes de fazer qualquer plano serio, tenho } \\
\text { de ter algum controlo na minha dor }\end{array}$ & 2,04 & 2,14 & 0,50 \\
\hline $\begin{array}{l}\text { CPAQ_18 - As minhas preocupações e receios acerca do } \\
\text { que a dor me irá fazer, são verdadeiros }\end{array}$ & 0,23 & 0,74 & 0,37 \\
\hline $\begin{array}{l}\text { CPAQ_4 - Sacrificaria com muito prazer coisas impor- } \\
\text { tantes na minha vida para controlar melhor esta dor }\end{array}$ & 2,99 & 1,67 & 0,05 \\
\hline $\begin{array}{l}\text { dor pode aumentar } \\
\text { docar-me em situaçoes nas quais a }\end{array}$ & 0,35 & 0,929 & 0,38 \\
\hline
\end{tabular}


Por outro lado, a escala total CPAQ apresentou uma correlação moderada e negativa com o Stress $(r=-0,501 ; p=0,000)$ e a Depressão $(r=-0,532$; $p=0,000)$, e uma correlação fraca e negativa com a Ansiedade $(r=-0,362$; $p=0,000)$.

A Realização de Actividades e a escala total CPAQ relacionaram-se de forma moderada e positiva com a Auto-Compaixão $(r=0,558$; $p=0,000 / r=0,535 ; p=0,000$, respectivamente). Para a Disponibilidade (CPAQ), foi obtido um valor de correlação fraco e positivo $(r=0,365$; $p=0,000)$. Os resultados mostraram igualmente correlações fracas e negativas entre as duas escalas da CPAQ, a Ruminação (RRQ10) e o Evitamento Experiencial (AAQ-II).

A importância da Aceitação da Dor, como factor preditor de psicopatologia, foi também analisada no âmbito do estudo da validade convergente da medida. Foram analisados os efeitos de colinearidade entre as variáveis independentes (através dos valores do Variance Inflaction Factor: VIF), a normalidade e homogeneidade dos resíduos (através do teste de Durbin-Watson e de representação gráfica). A inexistência de multicolinearidade entre as variáveis, homogeneidade e normalidade dos resíduos, permitiram a realização de análises de regressão linear múltipla. Seguindo os mesmos procedimentos dos autores da medida original, foram realizadas análises de regressão múltipla com selecção das variáveis stepwise, utilizando as sub-escalas da CPAQ (Realização de Actividades e Disponibilidade para a dor) como variáveis independentes e a Depressão, a Ansiedade e o Stress como variáveis dependentes. O Quadro 5 mostra os resultados das análises efectuadas.

A análise de regressão linear múltipla com selecção das variáveis stepwise, utilizando a Depressão como variável dependente, demonstrou que a Realização de Actividades contribuiu para $25,2 \%$ da variância deste sintoma $(\beta=-0,511 ; t=-5,475 ; p=0,000)$. Numa segunda análise de regressão linear múltipla com o mesmo método de selecção das variáveis e utilizando a Ansiedade como variável dependente, demonstrou que a Realização de Actividades foi, novamente a única sub-escala da $C P A Q$ que contribuiu para o modelo, explicando $11,4 \%(\beta=-0,353 ; t=-3,534 ; p=0,001)$. Finalmente, uma nova análise de regressão linear múltipla foi realizada, tendo o Stress como variável dependente, e mostrou que a Disponibilidade para a dor e a Realização de Actividades contribuíram 23,5\% para a explicação da variância do stress $(\beta=-0,271 ; t=-2,356 ; p=0,021)$.

\section{Validade Discriminante}

A validade discriminante da versão CPAQ obtida foi analisada através da capacidade de distinguir indivíduos com baixos ou elevados sintomas de depressão, ansiedade e stress (sub-escalas EADS-42). Foram formados dois grupos para cada sub-escala, recorrendo ao valor da mediana e 
utilizado o teste $t$ para amostras independentes. O Quadro 6 mostra os valores médios e os valores da estatística do teste, obtidos para a depressão, ansiedade e stress, separadamente.

Quadro 5. Preditores CPAQ da Depressão, Ansiedade e Stress

\begin{tabular}{|c|c|c|c|c|c|c|c|}
\hline V.D. & Passos & V.I. & $\overline{\mathbf{R}^{2}}$ & $\begin{array}{c}R^{2} \text { Ajus- } \\
\text { tado }\end{array}$ & $\overline{\mathbf{B}}$ & $t$ & $p$ \\
\hline Depressão & 1 & $\begin{array}{l}\text { Realização } \\
\text { de Activida- } \\
\text { des }\end{array}$ & 0,261 & 0,252 & $-0,511$ & 5,475 & 0,000 \\
\hline $\begin{array}{l}\text { \% Variância } \\
\text { Explicada }\end{array}$ & & & & $25 \%$ & & & \\
\hline Ansiedade & 1 & $\begin{array}{l}\text { Realização } \\
\text { de Activida- } \\
\text { des }\end{array}$ & 0,124 & 0,114 & $-0,353$ & $-3,534$ & 0,001 \\
\hline $\begin{array}{l}\text { \% Variância } \\
\text { Explicada }\end{array}$ & & & & $11 \%$ & & & \\
\hline Stress & 1 & $\begin{array}{l}\text { Disponibili- } \\
\text { dade para a } \\
\text { dor }\end{array}$ & 0,203 & 0,194 & $-0,295$ & $-2,568$ & 0,012 \\
\hline & 2 & $\begin{array}{l}\text { Realização } \\
\text { de Activida- } \\
\text { des }\end{array}$ & 0,253 & 0,235 & $-0,271$ & $-2,356$ & 0,021 \\
\hline $\begin{array}{l}\text { \% Variância } \\
\text { Explicada }\end{array}$ & & & & $24 \%$ & & & \\
\hline
\end{tabular}

Nota: V.D.= Variável Dependente; V.I.= Variável Independente

A Aceitação da Dor discriminou os indivíduos com mais e menos depressão. Assim, o grupo com mais sintomas de depressão apresentou menos realização de actividades $(M=30,33 ; D P=13,00)$ e menos disponibilidade para a dor $(M=12,52 ; D P=8.91)$, comparativamente com o grupo com menos sintomas de depressão $(M=43,25, \quad D P=10,87 ; \quad(t$ $(88)=-5,103 ; p=0,000) \quad / \quad M=20,79, \quad D P=11,58 ; \quad(t \quad(79)=-3,786$; $p=0,000)$, respectivamente). Para a ansiedade, foram encontradas diferenças nos grupos com mais e com menos sintomas no que diz respeito à realização de actividades $(t(91)=-3,750 ; p=0,000)$ e à disponibilidade para a dor $(t(86)=-3,362 ; p=0,001)$, no sentido do grupo com mais ansiedade relatar menos realização de actividades $(M=31,52 ; D P=12,05)$ e menos 
disponibilidade para a dor $(M=12,33 ; D P=9,33)$, em comparação com o grupo com menos ansiedade. Finalmente, as sub-escalas CPAQ discriminaram igualmente indivíduos com mais e menos sintomas de stress. Assim, foram obtidas diferenças entre os grupos para a realização de actividades $(t(88)=-2,992 ; p=0,004)$, no sentido do grupo com mais sintomas de stress revelar menos realização de actividades $(M=32,71 ; D P=13,88)$. Por outro lado, para a disponibilidade para a dor também foram obtidas diferenças entre os dois grupos $(t(89)=-4,395 ; p=0,000)$, com o grupo com mais sintomas de stress a relatar menos disponibilidade para a dor $(M=11,68$; $D P=9,47)$, comparativamente com o grupo com menos sintomas de stress.

Tabela 6: Diferenças entre os grupos com mais e menos sintomas de psicopatologia (depressão, ansiedade, stress).

\begin{tabular}{|c|c|c|c|c|c|c|}
\hline & \multicolumn{2}{|c|}{ Mais Depressão } & \multicolumn{2}{|c|}{ Menos Depressão } & \multirow[b]{2}{*}{$t$} & \multirow[b]{2}{*}{$p$} \\
\hline CPAQ & M & DP & $\mathbf{M}$ & DP & & \\
\hline $\begin{array}{l}\text { Realização de } \\
\text { Actividades }\end{array}$ & 30,33 & 13,00 & 43,25 & 10,87 & $-5,103$ & 0,000 \\
\hline $\begin{array}{l}\text { Disponibilidade } \\
\text { para a dor }\end{array}$ & 12,53 & 8,91 & 20,79 & 11,58 & $-3,786$ & 0,000 \\
\hline Escala total & 42,64 & 18,29 & 64,21 & 19,87 & $-5,271$ & 0,000 \\
\hline & \multicolumn{2}{|c|}{ Mais Ansiedade } & \multicolumn{2}{|c|}{ Menos Ansiedade } & & \\
\hline CPAQ & M & DP & M & DP & $t$ & $p$ \\
\hline $\begin{array}{l}\text { Realização de } \\
\text { Actividades }\end{array}$ & 31,52 & 12,05 & 41,36 & 13,24 & $-3,750$ & 0,000 \\
\hline $\begin{array}{l}\text { Disponibilidade } \\
\text { para a dor }\end{array}$ & 12,33 & 9,33 & 19,63 & 11,64 & $-3,362$ & 0,001 \\
\hline Escala total & 43,51 & 17,83 & 61,42 & 21,94 & $-4,250$ & 0,000 \\
\hline & \multicolumn{2}{|c|}{ Mais Stress } & \multicolumn{2}{|c|}{ Menos Stress } & & \\
\hline CPAQ & M & DP & $\mathbf{M}$ & DP & $t$ & $p$ \\
\hline $\begin{array}{l}\text { Realização de } \\
\text { Actividades }\end{array}$ & 32,71 & 18,88 & 40,98 & 12,10 & $-2,992$ & 0,004 \\
\hline $\begin{array}{l}\text { Disponibilidade } \\
\text { para a dor }\end{array}$ & 11,68 & 9,47 & 21,05 & 10,84 & $-4,395$ & 0,000 \\
\hline Escala total & 44,39 & 20,83 & 62,66 & 19,83 & $-4,176$ & 0,000 \\
\hline
\end{tabular}




\section{Discussão}

A investigação realizada teve como principal objectivo analisar a estrutura psicométrica da versão traduzida da CPAQ (McCracken et al., 2004) numa amostra de adultos portugueses com dor crónica. De facto, a conceptualização da ACT na base desta medida, reforça a importância da aceitação da dor como disponibilidade activa e consciente para experienciar pensamentos/ sentimentos/ sensações internas indesejadas, e o desinvestimento em torno de tentativas de controlar essas experiências (Bach \& Moran, 2008; Dahl et al., 2004; Hayes, 2005; Hayes et al., 2006; Hayes et al., 1999; Wicksell, et al., 2007). Para Hayes (2004), não pensar deliberadamente num pensamento, tende a fracassar, uma vez que a regra "não pensar acerca de X" contém o item a evitar (ou seja, "X"). Do mesmo modo, se é importante não se sentir ansioso, a ansiedade torna-se algo que provoca ansiedade.

No presente estudo, os resultados permitiram a realização de uma análise factorial em componentes principais. Foram respeitadas os parâmetros conhecidos da análise factorial efectuada, o método de rotação e a estratégia de selecção de itens, proposta por McCraken, et al. (2004). Foi identificada uma estrutura de dois factores, concordante com a versão da medida original, que explicou cerca de $51 \%$ variância do modelo. Na estrutura obtida, salientamos a existência de dois itens, que pesaram no factor respeitante à Realização de Actividades quando, na estrutura original, faziam parte do factor relativo à Disponibilidade para a dor (item 4 "Sacrificaria com muito prazer coisas importantes na minha vida para controlar melhor esta dor."; item 17 - "Evito colocar-me em situações nas quais a dor pode aumentar"). Por outro lado, o item 3 ("Não há problema em ter dor") pesou no factor relativo à Disponibilidade para a dor, quando na estrutura da medida original pertencia à Realização de Actividades.

No estudo de McCracken et al. (2004) foram obtidos alguns itens cujos pesos revelavam incongruências face ao factor de pertença, tendo os autores analisado individualmente os valores de consistência interna e os resultados dos factores, com e sem os itens discordantes. O mesmo procedimento foi realizado com os dados da nossa amostra, optando-se pela inclusão dos itens na escala de pertença na medida original, dado aumentar os valores de consistência interna e os valores de correlação item-total, dos dois factores.

A literatura de base sobre a medida original faz igualmente referência à estrutura de 4 factores (Realização de Actividades, Controlo do Pensamento, Cronicidade e Controlo da Dor), dois dos quais com fidelidade marginal (Controlo de Pensamentos e Cronicidade), e sem associações com a medida total. Estes factores não mostraram as relações esperadas com os indicadores de incapacidade, ansiedade e depressão (McCracken, 1998; McCracken et al., 2004; Vianne et al., 2003). 
Os resultados obtidos neste estudo são concordantes com as recomendações de McCracken et al., (2004) sobre a utilização da estrutura de dois factores, designados por Realização de Actividades (factor 1) e Disponibilidade para a Dor (factor 2). A análise dos dados efectuada é suportada pelos próprios resultados obtidos. Por outro lado, o poder explicativo desta organização dos dados é superior ao referido pelos autores da medida original.

Ao analisarmos os estudos realizados com esta medida, verificamos que a maioria utilizou a estrutura de dois factores da CPAQ (McCracken \& Eccleston, 2005; McCracken, Gauntlett-Gilbert \& Vowles, 2007; McCracken, MacKichan \& Eccleston, 2007; McCracken \& Samuel, 2007; McCracken \& Vowles, 2007; Morone, Greco \& Weiner, 2008; Nicholas \& Asghari, 2006). De facto, a conceptualização da Aceitação da Dor com dois componentes parece-nos de grande utilidade. A Realização de Actividades por parte do indivíduo com dor crónica tem implícito um processo mental que passa por um funcionamento diário positivo e funcional. Por outro lado, a disponibilidade face à dor passa pelo reconhecimento da ineficácia das estratégias de evitamento e de controlo, mas também pelo reconhecimento da cura como improvável (McCracken et al., 2004, Hayes et al., 1999; Viane et al., 2003).

No âmbito do estudo da validade convergente da medida, os resultados obtidos mostraram as relações esperadas com a psicopatologia. De facto, uma menor Aceitação da Dor relacionou-se com mais sintomas de depressão, ansiedade e stress, o que está de acordo com a literatura que salienta a preponderância deste conceito ao nível dos indicadores de funcionamento emocional, físico e social (Eccleston \& Crombez, 2007; McCracken, 1998; McCracken \& Eccleston, 2005; McCraken, Spertus, Janeck, Sinclair \& Wetzel, 1999; McCracken \& Vowles, 2007; Morone et al., 2008; Viane et al., 2003). Por outro lado, a Disponibilidade para a Dor e a Realização de Actividades, estiveram relacionadas com uma menor utilização de estratégias ruminativas, o que está de acordo com Viane et al. (2003). Estes autores mostraram que uma atitude de abertura face à experiência esteve na base da cessação de pensamentos intrusivos e aversivos.

As relações encontradas entre a Aceitação da Dor, a Auto-Compaixão e o Evitamento Experiencial forneceram, mais uma vez, suporte empírico para a aplicabilidade das abordagens baseadas na ACT no contexto da dor crónica (Bach \& Moran, 2008; Dahl et al., 2005; Hayes et al., 2006; Hayes et al., 1999; Hayes, 2004; Hofmann \& Asmundson, 2007; McCracken \& Eccleston, 2005; McCracken et al., 2004; McCracken \& Yang, 2006).

Incluído no estudo da validade convergente da CPAQ, foram ainda realizados procedimentos estatísticos de análise de regressão que confirmaram o poder preditivo da Aceitação da Dor na explicação dos sintomas de depressão, ansiedade e stress (McCracken, 1998; McCracken \& Eccleston, 2003; McCracken et al., 2004; McCracken et al., 1999; McCracken \&Yang, 
2006; Nicholas \& Asghari, 2006). O método de selecção das variáveis utilizado seguiu os procedimentos dos autores da medida original. A medida não tem um modelo subjacente definido, dado que McCracken et al. (2004) reforçam a existência de várias organizações factoriais, sendo algumas discutíveis, como referido anteriormente.

As duas sub-escalas CPAQ apenas foram retidas no modelo final do Stress, o que está de acordo com estudos que indicam um padrão de influência dos itens inconsistente, em função das variáveis em estudo (Depressão, Ansiedade ou Stress) (McCracken et al., 2004; Nicholas \& Asghari, 2006).

No que diz respeito à validade divergente, os resultados mostraram a capacidade das duas sub-escalas da Aceitação da Dor para discriminarem indivíduos com mais ou menos psicopatologia, o que reforça novamente a abordagem das duas dimensões implícitas à aceitação. A Realização de Actividades apesar da dor, implícita à Aceitação, pressupõe o reconhecimento da cura como improvável, e que a busca continuada de soluções e tratamentos são tentativas de evitar ou controlar a situação indesejada, conduzindo ao afastamento dos objectivos valorizados e do funcionamento saudável. Estes resultados reforçam uma vez mais a importância das abordagens centradas na $\mathrm{ACT}$, que ao possibilitarem ao indivíduo separar a experiência da dor do comportamento de evitamento, permitem simultaneamente experienciar a dor e viver uma vida valorizada (Hayes \& Duckworth, 2006; Hayes et al., 1999; McCracken \& Vowles, 2007; Wicksell et al., 2007).

Estes resultados parecem promissores para a população portuguesa com dor crónica, fornecendo alternativas inovadoras que se demarcam das abordagens tradicionais, centradas na redução do sintoma per se. Até ao momento, não são conhecidas intervenções baseadas na ACT na população portuguesa com dor crónica. Considera-se um campo de intervenção a privilegiar em futuras investigações, particularmente a adaptação da ACT a um caso jovem de artrite reumatóide. A investigação tem reforçado a importância das abordagens baseadas na ACT, pela possibilidade de promover a actuação do paciente em conformidade com objectivos e valores escolhidos, ao longo da experiência continuada de dor (Hayes \& Duckworth, 2006; Wicksell et al., 2007). Como situação específica de dor crónica, é particularmente relevante a análise da sensibilidade da CPAQ às mudanças da psicopatologia e do funcionamento físico neste tipo de pacientes.

Apesar dos resultados obtidos, este estudo apresenta algumas limitações. A análise da estrutura factorial da medida foi baseada nos escassos pressupostos referidos por McCracken et al. (2004). Os autores analisaram as distribuições das frequências dos itens, e os valores de correlação item-total. Numa primeira análise dos itens retiraram seis itens, por motivos empíricos ou por não apresentarem clara referência à dor. Ao referirem a análise factorial efectuada, não especificaram o método de selecção das 
variáveis nem os critérios de retenção nos respectivos factores. Obtiveram uma solução de quatro factores que referem como ineficaz, sendo reduzidas as informações fornecidas para aconselharem a utilização da estrutura com dois factores. Os autores referem os aspectos pelos quais retiram os dois factores menores, mas não fornecem alternativas ou sugestões para procedimentos adicionais. Outra limitação encontrada prende-se com as características da amostra utilizada pelos autores e da amostra portuguesa. Ambas as amostras foram, maioritariamente, constituídas por mulheres, casadas e reformadas. No nosso estudo, não registámos aspectos relativos à localização da dor. Os participantes com prevalência de dor há mais de seis meses foram referenciados pelo médico assistente, não sendo utilizado outro critério de inclusão no estudo. A maioria dos participantes dispunha apenas de tratamento farmacológico para a dor, não estando inserido em qualquer intervenção psicológica. Por outro lado, o estudo com a medida original não referencia a média de idades dos participantes, o que pode ser um aspecto relevante, uma vez que a Aceitação da dor como processo activo implica que o indivíduo mantenha um funcionamento adaptativo, participe em actividades agradáveis e actue em conformidade com os objectivos que estabeleceu para a sua vida, mesmo quando experiencia sensações indesejáveis. Neste sentido, a literatura em crianças e jovens com dor crónica é escassa, existindo poucas evidências empíricas sobre a eficácia das diferentes abordagens, onde a redução da severidade/ frequência da dor, não é o resultado mais importante (Eccleston, Morley, Williams, York \& Mastroyannopoulou, 2002; Wicksell, Melin \& Olsson, 2007).

Como abordagem desenvolvida no contexto comportamental do sofrimento humano, a $C P A Q$ introduz múltiplas inovações no estudo da cognição, da emoção e da mudança de comportamento. De facto, os resultados empíricos e os desenvolvimentos conceptuais na base desta medida possibilitam múltiplas vantagens em termos práticos. As terapias de terceira geração, em geral, e os modelos baseados na ACT, em particular, introduziram o conceito de flexibilidade psicológica nas abordagens psicológicas da dor crónica, permitindo desafiar directamente as sensações indesejáveis e dispor o indivíduo para a experiência álgica. $\mathrm{O}$ presente estudo parece assim justificar investigações adicionais centradas nos mecanismos de aceitação da dor, possibilitando desenvolver programas eficazes ao nível da promoção do funcionamento físico (dor e limitação) e psicológico (depressão e ansiedade).

\section{Referências}

Bach, P., \& Moran, D. (2008). ACT in practice. Case conceptualization in Acceptance \& Commitment Therapy. Oakland: New Harbinger Publications, Inc. 
Baer, R. (2006). Mindfulness-Based Treatment Approaches. Clinician's Guide to Evidence Base and Applications. Oxford: Elsevier Academic Press Publications.

Blackledge, J., \& Hayes, S. (2001). Emotion Regulation in Acceptance and Commitment Therapy. Psychotherapy in Practice, 57, 243-255.

Block-Lerner, J., Salters-Pedneault, K., \& Tull, M. (2005). Assessing mindfulness and experiential acceptance: Attempts to capture inherently elusive phenomena. In S. Orsillo \& L. Roemer (Eds.), Acceptance and mindfulness-based approaches to anxiety: Conceptualization and treatment (pp. 71-99). New York: Springer.

Burns, J., Kubilus, A., Bruehl, S., Harden, N., \& Lofland, K. (2003). Do Changes in Cognitive Factors Influence Outcome Following Multidisciplinary Treatment for Chronic Pain? A Cross-Lagged Panel Analysis. Journal of Consulting and Clinical Psychology, 71, 81-91.

Campbell-Sills, L., Barlow, D., Brown, T., \& Hofmann, S. (2006). Effects of suppression and acceptance on emotional responses of individuals with anxiety and mood disorders. Behaviour Research and Therapy, 44, 1251-1263.

Crombez, G., Eccleston, C., De Vlieger, P., Van Damme, S., \& De Clercq, A. (2007). Is it better to have controlled and lost than never to have controlled at all? An experimental investigation of control over pain. Pain. Retirada da web a 21 de Abril de 2008 de www.sciencedirect.com.

Dahl, J., Wilson, K., Luciano, C., \& Hayes, S. (2005). Acceptance and Commitment Therapy for Chronic Pain. Reno: Context Press.

Dahl, J., Wilson, K., \& Nilsson, A. (2004). Acceptance and Commitment Therapy and the treatment of persons at risk for long term disability resulting from stress and pain symptoms: A preliminary randomized trial. Behavior Therapy, $35,785-801$.

Dimond, A., \& Coniam, S. (1997). Controlo da dor crónica. Lisboa: Climepsi Editores.

Eccleston, C., \& Crombez, G. (2007). Worry and chronic pain: A misdirected problem solving model. Pain, 132, 233-236.

Eccleston, C., Morley, S., Williams, A., Yorke, L., \& Mastroyannopoulou, K. (2002). Systematic review of randomised controlled trails of psychological therapy for chronic pain in children and adolescents, with a subset meta analysis of pain relief. Pain, 99, 157-165.

Evers, A., Kraaimaat, F., Riel, P., \& Jong, A. (2002). Tailored cognitive-behavioral therapy in early rheumatoid arthritis for patients at risk: A randomised controlled trial. Pain, 100, 141-153.

Flor. H., \& Turk, D. (1988). Chronic back pain and rheumatoid arthritis: Predicting pain and disability from cognitive variables. Journal of Behavioral Medicine, $11,251-265$.

Gutiérrez, O., Luciano, C., Rodríguez, M., \& Fink, B. (2004). Comparison between an Acceptance-Based and a Cognitive-Control-Based Protocol for coping with pain. Behavior Therapy, 35, 767-783.

Hayes, S. (2004). Acceptance and Commitment Therapy, Relational Frame Theory, and the Third Wave of Behavioral and Cognitive Therapies. Behavior Therapy, $35,639-665$. 
Hayes, S., \& Duckworth, M. (2006). Acceptance and Commitment Therapy and traditional Cognitive Behavior Therapy approaches to pain. Cognitive and Behavioral Practice, 17, 185-187.

Hayes, S., Luoma, J., Bond, F., Masuda, A., \& Lillis, J.(2006). Acceptance and Commitment Therapy: Model, processes and outcomes. Behaviour Research and Therapy, 44, 1-25.

Hayes, S., Strosahl, K., \& Wilson, K. (1999). Acceptance and Commitment Therapy. An experiential approach to behaviour change. New York: The Guilford Press.

Hayes, S., Strosahl, K., Wilson, K., Bissett, R., Pistorello, J., Toarmino, D., et al. (2004). Measuring experiential avoidance: A preliminary test of a working model. The Psychological Record, 54, 553-578.

Hofmann, S., \& Asmundson, G. (2007). Acceptance and mindfulness-based therapy: New wave or old hat? Clinical Psychology Review. Retirada da web a 21 de Abril de 2008 de www.sciencedirect.com.

Jensen, M., Turner, J., Romano, J., \& Lawler, B. (1994). Relationship of pain-specific beliefs to chronic pain adjustment. Pain, 57, 301-309.

Lovibond, P., \& Lovibond, S. (1995). The structure of negative emotional states: Comparison of depression anxiety stress scales (DASS) with the Beck Depression and Anxiety Inventories. Behaviour Research and Therapy, 33, 335-343.

Maroco, J. (2007). Análise Estatística. Com utilização do SPSS. Lisboa: Edições Sílabo.

McCracken, L. (1998). Learning to live with pain: Acceptance of pain predicts adjustment in persons with chronic pain. Pain, 74, 21-27.

McCracken, L. (2006). Toward a full functional, flexible, and defused approach to pain in young people. Cognitive and Behavioral Practice, 13, 182-184.

McCracken, L., \& Eccleston C. (2003). Coping or acceptance: What to do about chronic pain? Pain, 105, 197-204.

McCracken, L., \& Eccleston, C. (2005). A prospective study of acceptance of pain and patient functioning with chronic pain. Pain, 118, 164-169.

McCracken, L., \& Eccleston C. (2006). A comparison of the relative utility of coping and acceptance-based measures in a sample of chronic pain sufferes. European Journal of Pain, 10, 23-29.

McCracken, L., Gauntlett-Gilbert, J., \& Vowles, K. (2007). The role of mindfulness in a contextual cognitive analysis of chronic pain-related suffering and disability. Pain, 131, 63-69.

McCracken, L., MacKichan, F., \& Eccleston, C. (2007). Contextual cognitive-behavioral therapy for severely disable chronic pain sufferers: Effectiveness and clinically significant change. European Journal of Pain, 11, 314-322.

McCracken, L., \& Samuel, V. (2007). The role of avoidance, pacing, and other activity patterns in chronic pain. Pain, 130, 119-125.

McCracken, L., Spertus, I., Janeck, A., Sinclair, D., \& Wetzel, F.(1999). Behavioral dimensions of adjustment in persons with chronic Pain: Pain related anxiety and acceptance. Pain, 80, 283-289.

McCracken, L., \& Vowles, K. (2007). Psychological flexibility and traditional pain management strategies in relation to patient functioning with chronic pain: An examination of a revised instrument. The Journal of Pain, 9, 700-707. 
McCracken, L., Vowles, K., \& Eccleston, C. (2004). Acceptance of chronic pain: Componente analysis and a revised assessment method. Pain, 107, 159-166.

McCracken, L., Vowles, K., \& Eccleston, C. (2005). Acceptance-based treatment for persons with complex, long standing chronic pain: A preliminary analysis of treatment outcome in comparison to a waiting phase. Behaviour Research and Therapy, 43, 1335-1346.

McCracken, L., \& Yang, S. (2006). The roles of values in a contextual cognitive-behavioral approach to chronic pain. Pain, 123, 137-145.

Morne, N., Greco, C., \& Weiner, D. (2008). Mindfulness meditation for treatment of chronic low back pain in older adults: A randomized controlled pilot study. Pain, 134, 310-319.

Neff, K. (2003). The development and validation of a scale to measure Self-Compassion. Self and Identity, 2, 223-250.

Nicholas, M., \& Asghari, A. (2006). Investigating acceptance in adjustment to chronic pain: Is acceptance broader than we thought?. Pain, 124, 269-279.

Öst, L. (2008). Efficacy of the third wave of behavioral therapies: A systematic review and meta-analysis. Behaviour Research and Therapy, 46, 296-321.

Pais-Ribeiro, J., Honrado, A., \& Leal, I. (2004a). Contribuição para o estudo da adaptação portuguesa das escalas de depressão ansiedade stress de Lovibond e Lovibond. Psychologica, 36, 235-246.

Pais-Ribeiro, J., Honrado, A., \& Leal, I. (2004b). Contribuição para o estudo da adaptação portuguesa das escalas de ansiedade, depressão e stress (EADS) de 21 itens de Lovibond e Lovibond. Psicologia, Saúde \& Doenças, 5, 229-239.

Pinto Gouveia, J., \& Costa, J. (2008). Questionário de Aceitação da Dor Crónica. Tradução e adaptação para a população portuguesa (manuscrito não publicado).

Pinto Gouveia, J., \& Castilho, P. (2006). Escala de Auto-Compaixão. Tradução e adaptação para a população portuguesa (manuscrito não publicado).

Pinto Gouveia, J., \& Gregório, S. (2007). Questionário de Aceitação e Acção-II. Tradução e adaptação para a população portuguesa (manuscrito não publicado).

Schiaffiano, K., Shawaryn, M., \& Blum, D. (1998). Examining the impact of illness representations on Psychological Adjustment to Chronic Illness. Health Psychology, 17, 262-268.

Segal, Z., Williams, J., \& Teasdale, J. (2002). Mindfulness-Based cognitive Therapy for Depression: A new Approach to Preventing Relapse. New York: The Guilford Press.

Smith, C., \& Wallston, K. (1992). Adaptation in Patients with Chronic Rheumatoid Arthritis: Application of a General Model. Health Psychology, 11, 151-162.

Tull, M., \& Gratz, K. (2008). Further examination of relationship between anxiety sensitivity and depression: The mediating role of experiential avoidance and difficulties engaging in goal-direct behaviour when distressed. Anxiety Disorders, 22, 199-210.

Turk, D., \& Okifuji, A. (2002). Psychological factors in chronic pain: Evaluation and Revolution. Journal of Consulting and Clinical Psychology, 70, 678-690.

Vowles, K., McNeil, D., Gross, R., McDaniel, M., Mouse, A., Bates, M., Gallimore, P., \& McCall, C. (2007). Effects of pain acceptance and pain control strategies on physical impairment in individuals with chronic low back pain. Behavior Therapy, 38, 412-425. 
Viane, I., Crombez, G., Eccleston, C., Poppe, C., Devulder, J., Van Houdenhove, B., $\&$ De Corte, W. (2003). Acceptance of pain is an independent predictor of mental well-being in patients with chronic pain: Empirical evidence and reappraisal. Pain, 106, 65-72.

Wicksell, R., Melin, L., \& Olsson, G. (2007). Exposure and acceptance in the rehabilitation of adolescents with idiopathic chronic pain - A pilot study. European Journal of Pain, 11, 267-274. 